\title{
Au rythme du discours proverbial
}

Rupture et continuité dans la parole ordinaire des Bwa (Mali)

The Rhythm of Proverbial Speech: Discontinuity and Continuity in Ordinary

speech among the Bwa (Mali)

\section{Cécile Leguy}

\section{(apenEdition}

\section{Journals}

\section{Édition électronique}

URL : https://journals.openedition.org/clo/2033

DOI : $10.4000 /$ clo.2033

ISSN : 2266-1816

Éditeur

INALCO

\section{Édition imprimée}

Date de publication : 1 janvier 2013

ISBN : 978-2-85831-217-7

ISSN : 0396-891X

Référence électronique

Cécile Leguy, «Au rythme du discours proverbial », Cahiers de littérature orale [En ligne], 73-74 | 2013, mis en ligne le 18 mai 2015, consulté le 01 juillet 2021. URL : http://journals.openedition.org/clo/2033 ; DOI : https://doi.org/10.4000/clo.2033

Ce document a été généré automatiquement le 1 juillet 2021.

\section{c) (1) (9)}

Cahiers de littérature orale est mis à disposition selon les termes de la Licence Creative Commons Attribution - Pas d'Utilisation Commerciale 4.0 International. 


\title{
Au rythme du discours proverbial
}

\author{
Rupture et continuité dans la parole ordinaire des Bwa (Mali) \\ The Rhythm of Proverbial Speech: Discontinuity and Continuity in Ordinary \\ speech among the Bwa (Mali)
}

\section{Cécile Leguy}

1 Tel qu'on peut le rencontrer dans les recueils de proverbes, le dire proverbial présente un aspect formel souvent assez particulier qui fait sa spécificité comme genre dit " figé » de la littérature orale, même si, en discours, le caractère figé des énoncés peut être remis en question par la variabilité de l'énonciation, ce que montre entre autres Anscombre (2005). À partir d'un corpus de cinq cents proverbes, il est possible de déterminer des formes récurrentes et un rythme propre au dire proverbial. Nombreux sont les essais de taxinomies formelles, reposant sur la structure (Milner, 1969; Barley, 1972 ; Dundes, 1975 ; Permyakov, 1979, pour ne citer que les plus connus), sur la syntaxe (Connena, 2000) ou sur la métrique du proverbe (Anscombre, 2000; Gouvard, 2006). Cependant, en situation, ce n'est pas toujours la forme qui fait le proverbe, celui-ci pouvant ne pas être prononcé intégralement, être tronqué ou même seulement évoqué par un mot ou quelques tons chantonnés entre les lèvres. En quel sens peut-on alors parler de rythme proverbial ? L'énonciation d'un proverbe impliquet-elle une rupture de rythme, ou bien faut-il distinguer les niveaux syntaxique et sémantique pour comprendre ce qui se joue lors de l'émission d'un énoncé proverbial? Faut-il nécessairement qu'un basculement ait lieu pour qu'on sache qu'un proverbe est émis?

2 Les énoncés dont il est question dans cet article ont été recueillis en situation auprès des $B w a^{1} d u$ Mali, agriculteurs sédentaires vivant à l'est du pays, à la frontière du Burkina Faso. Après une rapide présentation des aspects formels de la parole proverbiale telle qu'elle est observable dans ce contexte, nous verrons dans quelle mesure l'intervention d'un proverbe entraîne, ou pas, une rupture de rythme. 


\section{Aspect formel du proverbe : des constructions récurrentes}

3 Quelle que soit la langue dans laquelle ils sont émis, les énoncés proverbiaux répondent généralement à des contraintes formelles. Ils présentent une structure reconnaissable, qui rend repérable l'aspect proverbial d'un énoncé même quand on ne l'a jamais entendu. Il est possible, au sein d'un corpus de cinq cents proverbes bwa ${ }^{2}$, de chercher à relever les constructions récurrentes, d'un point de vue syntaxique. Trois grands types de structures dominantes peuvent être ainsi mis en valeur. Ne sera pas abordé ici l'aspect métrique ${ }^{3}$ des énoncés qui, même s'il n'est pas sans intérêt, nous éloignerait de notre propos.

\section{Premier type de construction proverbiale}

Une première structure formelle est repérable, non seulement à partir du recueil écrit des proverbes où ceux-ci sont présentés, en fin de volume, en liste alphabétique (Leguy, 1996, 321-332), mais aussi lors de l'énonciation. Ce premier type de construction est la forme majoritaire que prennent les énoncés proverbiaux en boomu, puisqu'elle concerne deux cent seize proverbes du corpus, soit $43,2 \%$. Elle met en forme une relation de cause à effet. Il s'agit d'une proposition causale, introduite par une expression du type : «si tu dis... » ('ò yí lo : tu/si/dis), « si tu vois... » ('ò yí màa : tu/si/ vois) ou "si tu autre verbe...", suivie d'une proposition principale, consécutive d'un point de vue logique, parfois explicitement introduite par : «alors... » (to).

Prenons pour exemple ce proverbe, souvent cité en début de discours, qu'un chef de famille pourra utiliser pour justifier le fait d'avoir convoqué tout le monde parce qu'il a une mise au point à faire: "Si tu vois une petite vieille courir dans les chaumes de fonio, si elle ne poursuit pas quelque chose, c'est que quelque chose la poursuit » ('ò yí màa hán-yíàzo, 'á lò lùi pè-nínnùù, lò yiì bò bé na, to bè bò la). Quand on a fauché le fonio, les tiges qui restent en terre sont très piquantes. La course est une activité propre aux enfants, et si l'on voit un adulte s'y adonner, il est évident qu'il se passe quelque chose de grave ou d'extraordinaire. Imaginons une petite vieille, comme celles que l'on a coutume de ne rencontrer qu'au fond des villages, occupées à filer du coton en causant tout en mâchant du tabac; une de ces vieilles qui n'ont guère l'habitude de porter des chaussures et ne vont plus dans les champs depuis que leurs jambes sont devenues si maigres. Imaginons cette petite vieille courir ainsi tel un simple bambin. Si cela se réalise, c'est qu'il se passe effectivement quelque chose de grave. En énonçant, à l'initiale de son discours, ce proverbe introductif présentant cette image, le patriarche met en valeur l'importance de ce qui le pousse à convoquer une réunion de famille.

6 Si un rythme spécifique est ici introduit du fait de la formulation même du proverbe, signalée par ces simples mots "si tu vois...», la rupture est également d'ordre sémantique, ce qui est caractéristique du discours proverbial. Pour qu'il y ait proverbe en effet, il faut un transfert de sens, ce qui est bien signifié en boomu où " proverbe » et « devinette » (jeu de questions-réponses) sont désignés par un même terme, wàwe, les deux pratiques langagières présentant la caractéristique commune de faire deviner ce qui doit être entendu derrière les mots énoncés (Leguy, 2004). 
7 Ainsi, lors de l'émission d'un proverbe, ce n'est pas seulement la structure formelle qu'il présente qui vient rompre le cours de l'énonciation. La rupture se fait également du point de vue sémantique, l'image d'une vieille courant à travers champ ou le récit des déboires d'un animal venant interférer avec les propos plus explicites de l'énonciateur. Prenons un exemple de conversation : des villageois discutent au sujet du mariage d'une fille du village. Un étranger qui assiste à la discussion demande la parole pour donner son point de vue.

ÉTRANGER : « Si tu donnes trop de conseils à une fille dans le choix de son futur mari, tu prends le risque de conclure un mariage sans réel avenir pour elle.

('ò yí bé hánzúnú má bè lo léra mi sào báró, ò yíca-sí, à lo cé yamu ne hìró mána).

VILLAGEOIS 1 : Tu connais les filles de chez toi, mais tu ne connais pas les filles de chez

nous!

('úé zún mi lóo-hánzáwé bè ma wùró, 'a ò bècézún wàbé lóo-hánzáwé bè).

VILLAGEOIS 2 (s'adressant à villageois 1 avec un proverbe) : - Si tu ne veux pas qu'un

enfant mange de tes poules de sacrifice, ne les lui donne pas à plumer ».

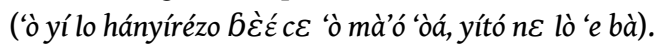

$8 \mathrm{Du}$ point de vue syntaxique, on peut remarquer que la construction proverbiale s'inscrit finalement assez bien dans la manière ordinaire de parler, où il est d'usage comme on peut le voir dans les propos de l'étranger de recourir au pronom personnel de la deuxième personne du singulier en tant qu'indéfini. Si l'on reconnaît le proverbe de par sa formulation en "si... alors ", la rupture induite est d'abord sémantique. Cet énoncé met également en scène une relation de cause à effet, formulée comme une recommandation, ce qui est très fréquent dans le discours proverbial. Ici en l'occurrence, le deuxième villageois signale au premier que, dans la mesure où l'on a accepté de discuter de ce sujet devant l'étranger et qu'on lui donne la parole, on ne peut pas lui reprocher ensuite d'émettre un avis. Ce proverbe est ainsi énoncé pour signifier qu'il faut savoir assumer les conséquences d'une promesse ou d'un engagement. Lorsqu'on demande un travail à un enfant, il est en droit de réclamer quelque chose en récompense. Puisqu'on prétend que la consommation de la viande utilisée pour les sacrifices rendrait l'enfant stérile, il vaut mieux éviter d'avoir à lui en donner un morceau. Il faut donc réfléchir aux conséquences avant de s'engager.

Nous n'entrerons pas dans les détails en explorant plus avant les différents types structuraux que peuvent prendre les énoncés présentant cette construction en «si... alors ». Notons seulement que le raisonnement logique de l'implication («si p, alors q" ou $« p \rightarrow q »)$, semble bien être une manière privilégiée pour le dire proverbial (GomezJordana Ferary, 2012, 56 et suivantes). Il est remarquable que ce premier type concerne une très forte majorité des énoncés du corpus (sans compter les variantes de proverbes recueillis sous une autre forme).

\section{Deuxième type de construction proverbiale}

10 La deuxième construction récurrente concerne quarante-six proverbes du corpus (soit 9,2\%). Il s'agit de la formule commençant par "C'est...» ('a...) qui pose une évidence à valeur de vérité universelle (à l'instar des formules en «qui » du corpus français). Ainsi l'énoncé proverbial suivant: alors qu'il sort rarement de chez lui, le vieux Wari participe ce jour-là aux festivités et va d'une maison à l'autre, offrant de la bière de mil à ceux qui l'entourent partout où on en vend. Les gens s'étonnent : 
VILLAGEOIS 1: «Moi, j'ai vu Wari par là-bas tout à l'heure, le voici encore qui passe par ici.

( $m \varepsilon$ fo màa Wárí má hè vé, déle ja 'à 'ànín má hè).

VILLAGEOIS 2 :-C'est vrai ! Moi aussi je l'ai vu. Il était en train d'acheter de la bière pour en donner à des gens. Je l'ai vu trois fois le faire.

( $m u$ bó ! $m \varepsilon$ múso màa lo, 'a lo có nàn 'a han nìin ; 'un màa lo tuii cuá tiin, 'a lo wée mu).

VILLAGEOIS $3:-C$ 'est la volonté du vautour qui courbe son bec ».

('a dùba sà'o wée 'ùarí lo nùbwo).

11 Autrement dit, pour une fois qu'il sort de chez lui, il le fait de sorte que tout le monde le voie, tout comme l'acharnement que le vautour manifeste sur toutes sortes de charognes finit par se voir à la forme de son bec. Ce proverbe peut également être énoncé d'une autre manière : «C'est la volonté du vautour qui lui écorche la tête (qui le fait chauve) » ('a dùba sà'o wée 'ùe lò jun). D'un point de vue purement logique, on est également ici face à une implication («c'est $p$ qui q » est aussi équivalent au « $p \rightarrow q$ » de la logique des propositions). Dans ce genre de proverbes, une caractéristique d'un personnage ou d'un animal est mise en avant pour énoncer une proposition à valeur de vérité absolue. Ici, le vautour est remarquable d'une part du fait de la forme de son bec, d'autre part par sa calvitie, deux attributs qui semblent faire partie de l'essence même de l'être-vautour. Ces proverbes ou des énoncés du même type seront utilisés lorsqu'un acte ou un fait se révèlera être une conséquence directe de l'identité de la personne concernée. L'émission du proverbe, repérable à sa formulation en 'a («c'est») lui donnant un aspect sentencieux, entraîne une rupture - syntaxique et sémantique dans le cours de la conversation. D'autres formules construites sur le même modèle présentent de manière plus anodine une vérité universelle, par exemple «C'est petit à petit que la tête du cochon grossit » ('a dédéc wée de sio jun wa), équivalent du proverbe français « Petit à petit, l'oiseau fait son nid ».

\section{Troisième type de construction proverbiale}

On rencontre aussi un certain nombre de proverbes présentant deux actions incompatibles (trente-et-un proverbes de ce corpus, soit 6,2\%). L'énoncé concerne un personnage indéterminé sous le nom de « quelqu'un » (nùu) ou «tu » ('ò), le pronom de la deuxième personne du singulier désignant ici encore l'indéfini. C'est en général par

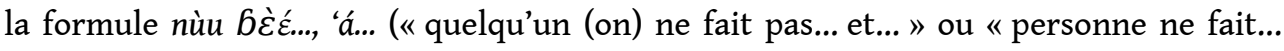
et... ») que l'on rencontre ce type d'énoncé proverbial. Ainsi dans le proverbe suivant : «On ne peut pas aller aux fiançailles du varan et déclarer qu'on ne rentrera pas dans

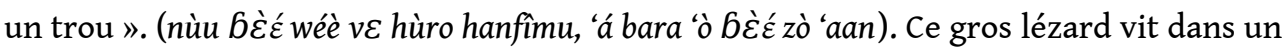
terrier et on n'imagine pas participer à une réjouissance le concernant sans lui faire l'honneur de pénétrer dans son domicile. Il faut se situer ici dans un contexte ouestafricain où la visite d'un ami ou d'un étranger est conçue comme une bénédiction. Pourrait-on participer à une fête sans aller chez la personne concernée? Comment prétendre résoudre cette contradiction? Ce proverbe sera émis à l'adresse de quelqu'un qui agit de façon contradictoire ou qui prétend vouloir faire des choses incompatibles. Les énoncés de ce type mettent ainsi généralement en scène deux actions qui ne peuvent pas être menées ensemble ou deux choix obligés, que l'on peut ainsi traduire en logique des propositions : « $\neg \mathrm{p}^{\wedge} \mathrm{q} »$, la première proposition étant généralement négative, la seconde ajoutant le contexte qui justifie cette négation. « On ne peut pas frapper la tempe et présenter ses excuses à l'oreille » (nùu bè ć cá sàbuá, 'á 'ò bara júvànlo fé 'àféré) si proches qu'on ne peut toucher l'une sans l'autre - dit-on par 
exemple pour inciter une personne à assumer les conséquences de ses actes, tout comme on fera taire celui qui parle sans preuve en lui disant : " On ne peut pas se tenir dehors et connaître le chef des morts ». (nùu bèź wée 'En 'iin, 'aà zuna nùhuora-be). Tant que l'on n'a pas fait l'expérience de la mort, on ne peut rien dire de ce monde dont on ne connaît rien, pas même le chef. «Tu n'as pas vu la fiancée et tu étendrais la natte ?» (nùu 6 č ć màa hánfían, 'á 'o $6 \varepsilon$ be zánlé), dit-on encore pour inciter à la prudence.

13 Ces trois constructions sont les plus courantes et nous voyons que chacune de ces formes correspond à un mode de raisonnement particulier. La première met en jeu une implication logique du type relation de cause à effet, la deuxième une évidence à valeur universelle et la troisième l'incompatibilité de certaines actions, les trois ayant en commun une structure binaire. On peut aisément comprendre pourquoi ces formes sont privilégiées dans l'émission d'un énoncé qui prend souvent une valeur argumentative. On peut par ailleurs avancer que la construction d'un énoncé n'est pas négligeable d'un point de vue strictement formel et qu'il entre en ligne de compte tant dans la réussite de l'émission du proverbe que dans sa mémorisation.

\section{Énonciation d'un proverbe et rupture de rythme}

Outre ces trois types structuraux repérables au sein du corpus, deux autres caractéristiques formelles sont notables dans la mesure où elles entraînent une rupture de rythme dans le discours au moment de leur énonciation. Il s'agit, d'une part, du rythme binaire qui qualifie de nombreux proverbes, d'autre part du fait que l'énoncé soit parfois mis dans la bouche d'un animal ou d'un personnage, posant acte de la citation par une formule qui introduit le proverbe sous forme de discours rapporté.

\section{Le rythme binaire}

15 Le rythme binaire a souvent été avancé par les parémiologues comme déterminant dans la composition de l'énoncé proverbial (Jolles, 1976; Anscombre, 2000). Il est en effet remarquable que l'on puisse retrouver une structure binaire dans la majeure partie des proverbes du corpus. La binarité peut aussi jouer un rôle d'indicateur: le proverbe au rythme scandé sur deux temps vient rompre le rythme plus anarchique du discours et nous signaler ainsi sa présence.

16 Si les exemples précédents peuvent être analysés dans une certaine mesure comme présentant un rythme binaire, puisque chaque proverbe comporte deux parties plus ou moins marquées, cet aspect est plus fortement présent dans l'émission de formules dont la rythmique semble particulièrement travaillée, du fait de la brièveté des deux parties. Ce proverbe que l'on pourrait ainsi traduire en français : « Du bœuf que l'on va tuer demain, on ne mesure pas les cornes " (nà ne 6wé hiró, hwa bèć wéè màzí), est un bon exemple. Agencés autrement - on peut par exemple imaginer qu'on puisse dire, selon la première construction notée comme récurrente: «Si tu dis que tu vas tuer le bœuf demain, tu n'en mesures pas les cornes ", ou bien selon la troisième qui pourrait également convenir: "On ne mesure pas les cornes d'un bœuf qu'on va tuer le lendemain »- les mots de ce proverbe n'auraient pas eu le même impact que l'aspect sentencieux dû au rythme bien scandé de la formule canonique. Cet énoncé présente en outre un isosyllabisme (les deux parties du proverbe comptant cinq syllabes chacune), procédé également remarqué dans de nombreuses langues comme marquant la 
«structure rythmique» du proverbe (Anscombre, 2000,19). Si cette caractéristique n'est pas généralisable concernant le présent corpus, elle est cependant remarquable dans des énoncés au rythme bien balancé, à l'instar de celui-ci. Sans être à proprement parler spécifique au dire proverbial, la construction par juxtaposition, faisant l'économie de toute conjonction, accentuant ainsi d'un point de vue syntaxique la binarité rythmique, est également observable dans certains proverbes du corpus, comme on peut le voir dans l'exemple précédent ou bien dans le suivant: "Ce qu'on fait à l'essaim de trigones [mouches à miel], on ne saurait le faire à la ruche » (biò mà wá yíyo, 6 غ̇é wá fán).

17 Tous les proverbes recueillis qui présentent ainsi une structure binaire remarquable du fait de leur brièveté, de l'isosyllabisme de leurs parties ou du recours à la juxtaposition, sont généralement bien connus et très souvent employés. Lors des enquêtes, la valeur rythmique de ces énoncés a toujours été avancée pour justifier le fait qu'ils soient privilégiés. Certains proverbes sont même valorisés parce qu'on leur reconnaît un rythme proche du chant du tambour. C'est le cas par exemple du proverbe suivant: "Ce que tonnerre foudroie, feu le brûle » (wúro nà bè nE, dán za bún).

Le tempo de l'énoncé en permet une meilleure mémorisation qui vient s'ajouter au plaisir de dire une formule qui sonne bien, dont le rythme renforce le sens en lui donnant un appui phonique non négligeable.

\section{Le cas des proverbes ayant un auteur spécifié}

De nombreux proverbes sont introduits par un personnage qui parle et énoncés comme tels. Il s'agit de "dits d'animaux », parfois aussi de végétaux, de divers personnages particuliers tels le griot ou le fantôme, ou bien encore de formules attribuées à un ancien réputé pour ses bons proverbes. La formule introductive : « $\mathrm{X}$ a dit que...» ( $\mathrm{X}$ lo...) qui met ainsi le proverbe dans la bouche de "quelqu'un d'autre " que le seul énonciateur ${ }^{4}$, interpelle en signalant explicitement qu'un proverbe va être dit, que le bon mot d'un personnage particulier va être à l'instant rapporté parce qu'il convient exactement à la situation actuelle qu'il saura éclairer. Cette présentation sous forme de citation signale le proverbe comme tel. Selon Chambat-Houillon et Wall, «Citer, ce n'est pas uniquement répéter les mots d'autrui, c'est les reprendre avec le but de faire un clin d'œil sémantique à quelqu'un d'autre, avec un désir d'illustration aussi » $(2004,11)$. Le fait même d'être dits comme des citations introduit une rupture, non seulement sémantique (on entend d'un coup parler un animal), mais aussi syntaxique et énonciative de par l'introduction de termes signifiant qu'il s'agit bien d'un discours rapporté : on entend en quelque sorte "une autre voix». Cent vingt proverbes ${ }^{5} \mathrm{du}$ corpus sont ainsi énoncés par un animal, un végétal ou un personnage. C'est le cas de : "L'écureuil fouisseur dit: "c'est le froid (qu'on attrape à l'endroit) de la causette qui te poursuit au lit” " (báho lo : 'a nùu sunmúnu-tanú wéè da má 'ó). Il s'agit d'un écureuil qui craint le froid et rentre dans son trou dès le coucher du soleil. Cette caractéristique met l'accent sur sa prévoyance, et c'est elle que l'on retient dans ce proverbe pour signifier que les conséquences d'une action ou d'un événement peuvent se faire sentir encore bien après son achèvement. Le recours à un animal précis (ou à un personnage...) est toujours significatif et l'énoncé est mémorisé comme tel, en tant que « dit d'animal ».

Si les proverbes de ce type ne sont pas tous issus d'un conte, ils font cependant souvent appel à un animal bien connu de ce genre narratif ou bien à un animal familier dont les 
caractéristiques - physiques ou comportementales - évidentes sont immédiatement comprises de tous. Certains sont plus précisément extraits d'un conte ou d'une fable ils en reprennent généralement la fin - et c'est alors non seulement la formule, mais aussi toute l'histoire qui est sollicitée, qu'il est nécessaire de connaître pour comprendre l'allusion et accéder au sens des propos émis. Par exemple, un homme ayant aidé quelqu'un qui était dans le besoin et semble l'ignorer depuis que sa situation est meilleure, peut adresser à l'ingrat - ou en parlant de lui - le proverbe suivant: «Sa'oui (la hyène des contes) dit : vraiment, Ba'oura (le petit bouc) est devenu grand !» (sáuìi lo : ba'ùrà màà 'a yàró dé!). Il est difficile de comprendre ce qu'il sous-entend si on ne connait pas le récit dont est issue cette phrase, devenue proverbe. Voici résumée la fable en question :

Sa'oui-la-hyène se promenait en compagnie de Sinizo, le petit lièvre, lorsqu'ils aperçurent un scorpion que Sa'oui, toujours affamée et croyant voir, dans cet être «cornu », un petit bouc, voulut croquer sur le champ. Sinizo la retint en lui disant que l'animal était bien petit pour être intéressant à manger. Ils reprirent leur promenade, mais Sa'oui crut ruser en abandonnant son bonnet, qu'elle prétendit ensuite avoir oublié, délaissant le petit lièvre pour revenir vers l'arbre et engloutir le scorpion. Bien entendu, elle se fit méchamment piquer le nez et rejoignit piteusement le petit lièvre. Quelque temps plus tard, rencontrant Ba'oura-le-petitbouc, elle s'enfuit à toutes jambes au souvenir de cette piqûre, en s'exclamant «Vraiment, Ba'oura est devenu grand!»

21 La rupture de rythme introduite par l'émission du proverbe est morphologique, avec l'introduction du discours rapporté de la hyène, mais aussi sémantique et énonciative, car ce ne sont pas seulement les mots qu'à l'écrit on mettrait entre guillemets qui sont invités, mais aussi tout le déroulement du récit qu'il faut visualiser pour saisir l'allusion. En effet, la relation entre Sa'oui et Ba'oura mise en scène dans le proverbe est la conséquence d'une relation antérieure explicitée dans la fable, qu'il est nécessaire de connaître si l'on veut comprendre cette histoire d'animaux qui interrompt le discours. L'aventure que Sa'oui a eue avec le scorpion influence son attitude face au petit bouc. Pour elle, le scorpion qu'elle voit au départ est l'équivalent d'un petit bouc, c'est-à-dire de quelque chose d'intéressant à manger et de facile à attraper. Or, quand elle essaye de le dévorer, elle se fait piquer. Quand plus tard elle rencontre le bouc, elle pense faire preuve d'intelligence en déduisant qu'elle pourrait en recevoir une grande piqûre, comme s'il était un grand scorpion. Elle choisit donc de prendre la fuite. L'erreur de la hyène est en fait de ne pas avoir compris qu'il existait deux sortes d'animaux, ceux qui piquent d'une part, les inoffensifs d'autre part, et d'assimiler ces deux catégories, en ne distinguant que la taille. N'oublions pas que la hyène a pour attributs bêtise et couardise! Le cheminement de la fable est donc nécessaire à la compréhension du proverbe, qui n'a retenu que la fin du raisonnement, que la conséquence. Dans cette situation, on peut dire que, ironiquement, le diseur de proverbe donne à son interlocuteur en même temps le rôle du petit bouc qui a grandi et de la hyène qui le constate. Car si celui-ci a "grandi » en richesse au point de se permettre d'ignorer un ancien bienfaiteur, c'est lui-même qui s'illusionne en pensant être devenu quelqu'un d'important. C'est l'erreur de jugement de Sa'oui - et sa stupidité par la même occasion - qui est signifiée ici dans l'emploi du proverbe.

Il arrive aussi fréquemment qu'un proverbe soit énoncé comme une citation d'auteur, introduit par le nom de son créateur et celui de son village. Par exemple, peut-on entendre dire : "Batchiri de Yasso dit que trop de mise en commun mène à la ruine " (yá'ui bacírí lo : párí sùme wéè tèni nùu), ce personnage, Batchiri du village de Yasso (yá'ui 
en boomu), ayant à son actif plusieurs énoncés célèbres. Par ailleurs, il arrive dans certains villages que l'on accorde à un personnage, bien connu de tous pour son habileté à manier les proverbes, des énoncés qui peuvent être cités sans introduction ailleurs. Dans le « Répertoire d'Augustin Dembélé de Wara » comprenant cent quatrevingt-huit proverbes recueillis par son fils, Alexis Dembélé (non édité, documentation personnelle), on remarque que tous les énoncés sont attribués à une personne, homme ou femme, du village de Wara ou des alentours, dont des énoncés connus sans introduction ou bien mis dans la bouche d'un animal. Augustin ne cite pas de proverbe sans l'attribuer à un bon diseur de sa connaissance! Il arrive aussi parfois qu'on introduise son dire proverbial par un appel aux anciens ou plus largement à la communauté tout entière, en rappelant que « les Bwa disent... ", référence normative qui signale la présence du proverbe tout en donnant plus de poids au discours personnel de l'énonciateur.

Ces formules introductives, qu'elles attribuent l'énoncé à un animal, à une personne ou bien à la communauté tout entière, sont en situation d'énonciation une manière d'amorcer le passage au discours proverbial et introduisent de fait un basculement de rythme en créant une rupture. Ainsi que le rythme binaire, plus ou moins marqué, qui caractérise la plupart des énoncés proverbiaux, elles sont une manière de signaler, à l'intérieur du discours, ce qui doit être entendu comme une "parole forte» (les Bwa disent une « voix/tanu »), ce qu'il faut retenir.

\section{Usage de la contraction, du télescopage...}

En boomu, on aime également user des contractions. Cela se retrouve plus particulièrement dans la composition de noms propres et, bien entendu, dans le discours proverbial qui est alors encore plus percutant. Il faut parfois déplier complètement une formule pour réussir à comprendre ce qu'on a voulu dire, tant les syllabes et la tonalité de la phrase se sont télescopées. Cette façon de contracter mots et tons est d'autant plus privilégiée dans les formules proverbiales qu'elle permet de dire beaucoup de choses de la manière la plus courte possible, une phrase entière pouvant se voir énoncée en condensé par un seul vocable inventé pour l'occasion. Le proverbe n'est plus alors seulement un énoncé qui vient poser ses images sur la réalité, il s'impose en bousculant le discours par la force de sa concision.

Ainsi, certains proverbes présentent des éléments télescopés que celui qui maîtrise la langue comprend immédiatement, mais qui restent bien obscurs au néophyte. Prenons un exemple : " "Kapokier-rien”, et s'il commence à se faner? (dò'óró-6we'e, 'a hini 'a lì yi so'osa'a lé ?). Pour vraiment comprendre ce qui se cache derrière cette formule lapidaire, il faut en quelque sorte la déplier pour retrouver les mots dissimulés dans le proverbe, des mots qui, s'ils étaient prononcés, feraient perdre tout son poids à cette phrase. L'énoncé peut ainsi être découpé en quatre parties, la première "dòóró- $6 w e$ 'e » et la quatrième "so'osa'a lé» se répondant, tandis qu'au centre se suivent " 'a hini » et " 'a lì yi ». dò'óró- $b w e$ 'e est une expression créée pour l'occasion en assemblant le nom d'un arbre, le kapokier, au terme « 6 we'e » que l'on peut traduire par « rien » ou " pour rien ». Il faut comprendre ici qu'il s'agit du kapokier commun, de cet arbre fragile au bois tendre qui est déjà en perpétuel danger, car non protégé par la coutume contrairement à d'autres arbres tels le karité ou le balanzan - et que l'on n'hésite pas à ébrancher ou à abattre pour faire de petits bancs. Cet arbre donne de belles fleurs 
rouge-orangé de décembre à février, et on en utilise le calice pour confectionner une sauce que les Bwa apprécient beaucoup avec le tô (flan de mil). «Quand il commence à se faner " est la période où l'on ramasse ses fleurs, et à cette fin on n'aura aucun scrupule à le dépouiller de ses branches ou même à l'achever pour que la cueillette soit plus facile. On pourra dire ce proverbe au sujet d'un jeune qui est connu pour sa paresse et qui vient de se blesser la jambe : lui qui, d'ordinaire, n'est déjà pas très productif quand il travaille aux champs, c'est la catastrophe!

Les proverbes suivants doivent aussi leur rythme à la concision de leur formule dont les mots sont combinés, voire amalgamés, cette formulation raccourcie et quelque peu obscure faisant de ces proverbes des énoncés privilégiés par les vieux diseurs de bonnes paroles: "Arracher-planter" n’a pas de racine" (còó $f \mathcal{E}$ cinù mána), dit-on communément au sujet de l'étranger qui n'est plus chez lui nulle part, lui attribuant un nom (còó-fE) qui résume en deux mots sa situation. On retrouve ici en effet une forme de construction souvent utilisée dans la composition des noms propres de personne ou de chien: une combinaison de verbes ou un ensemble syntagme nominal-syntagme verbal associé du point de vue phonologique et tonologique qui dépasse rarement trois syllabes. Les proverbes présentant ce type de contraction permettent de s'adresser directement à quelqu'un en lui donnant une identité significative (Leguy, 2006). Ainsi, l'interlocuteur se voit attribué un nouveau nom propre inventé pour l'occasion, nom porteur d'un message qui lui convient dans la situation présente, comme l'illustre le proverbe suivant : “"Si j'avais su" vient en dernière position » (yáwé-zun, dú ma bènE), proverbe qu'on peut adresser à celui qui tarde trop à se décider, pour le mettre en garde d'avoir des regrets plus tard. Ou encore cet énoncé, entendu lors d'une discussion entre deux frères sur la segmentation éventuelle de la grande famille en foyers autonomes: "Les parents de "je ne suis pas concerné" ${ }^{6}$ ne récoltent pas beaucoup de

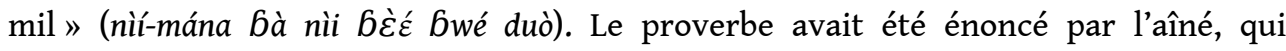
souhaitait que la vie familiale communautaire persiste malgré toutes les difficultés que rencontraient les frères à travailler ensemble. Le personnage imaginé, au nom évocateur, rappelait celui qui se désintéresse de la famille au profit de son propre intérêt et qui en fait pâtir tout le monde.

Présenté en tant que forme achevée d'une bonne manière de parler qui privilégie les sous-entendus et les constructions poétiques, on comprend que le dire proverbial recherche et valorise ces manières de dire en raccourci qui rappellent la façon dont sont composés les noms propres. La formulation elliptique, faisant l'économie de certaines syllabes, déplaçant le ton d'un phonème occulté sur celui qui le précède, permet ainsi en très peu de phonèmes d'évoquer toute une partie de la phrase, tout comme dans les deux ou trois syllabes qui composent un nom propre peut se glisser toute une histoire. Du point de vue de l'énonciation, le rythme rendu plus rapide que si le propos était développé n'en est que plus percutant, incitant à prêter attention à ce qui a été évoqué. S'il arrive qu'on rencontre le même proverbe formulé de différentes façons, la variante la plus contractée est généralement considérée comme la meilleure, la plus percutante, la mieux rythmée. Dans le proverbe suivant, par exemple, la fin de l'énoncé est elliptique et semble résumer une action qui reste sous-entendue : 'ò yí màa 'á sáuùi ma yîa má vizà-Jun'ué, to bè wón. Si l'on traduit directement ce proverbe en français, on obtient une phrase qui se termine étrangement : «Si tu vois que la hyène s'amuse avec des têtes de chevreaux, alors "la chose est faite" ». Dans une variante du même proverbe, nous retrouvons une forme plus explicite pour le traducteur, de la consécutive : 'ò yí màa 'á sáuìi ma yỉa má vizà- nun'ué, to he mi lo sían jun-sé mana ${ }^{8}$ (« Si tu 
vois que la hyène s'amuse avec des têtes de chevreaux, alors on ne peut pas dénombrer celles qui sont déjà dans son ventre »). Ici les choses sont dites clairement, mais cette deuxième formule plus longue est aussi beaucoup moins percutante, le rythme s'y épuise avant la fin de l'émission, et c'est la première formule qui fera l'unanimité des meilleurs diseurs de proverbes. Ainsi, on trouve dans la première formulation une proposition finale à trois syllabes, tandis que la première partie du proverbe est longue de quinze syllabes. Cette deuxième partie à trois syllabes rapproche l'énoncé de proverbes plus courts et plus cinglants, comme « Ce qu'on fait à l'essaim de trigones, on ne saurait le faire à la ruche " (biò mà wá yíyo, 6 ċé wá fán) ou "Ce que tonnerre foudroie, feu le brûle » (wúro nà bè ne, dán za bún) vus plus haut.

À partir des différents exemples de formulation présentés ici, nous pouvons constater la présence d'éléments qui font la pertinence du proverbe et qui font qu'on le reconnait comme tel: le respect d'une structure logique, la binarité, le recours à un discours rapporté dont l'auteur est cité, la présence de formulations raccourcies... Il est ainsi possible de noter ce qui dans la forme donne son caractère de " proverbe » à un énoncé. Mais le proverbe tel qu'on peut le connaître ou le reconnaître est une chose, l'énoncé du proverbe en situation en est une autre.

\section{Le proverbe en situation : rompre ou ne pas rompre le rythme?}

29 L'observation des échanges langagiers au cours desquels sont dits des proverbes permet de faire quelques remarques, qui obligent à relativiser l'importance à accorder à la forme du proverbe et à s'interroger sur la nécessité d'un basculement de rythme dans le dire proverbial.

\section{La forme ne suffit pas à faire un proverbe}

Qu'est-ce en effet qu'un proverbe? Dans quelle mesure le rythme définit-il le genre proverbe? Est-ce la forme d'un énoncé qui permet de classer celui-ci dans une catégorie prédéfinie comme étant le dire proverbial? Ou bien la dimension métaphorique, entraînant une rupture sémantique, est-elle ce qui détermine en premier lieu le discours proverbial?

31 Le recueil d'énoncés en situation conduit à faire la remarque suivante. Il est des énoncés qui ont la forme d'un proverbe, reprenant une des constructions récurrentes notées plus haut, et qui pourtant ne seront pas considérés comme des proverbes lors de leur émission. Par exemple lors d'une discussion au sujet de problèmes financiers, l'un des interlocuteurs a dit la phrase suivante : «Si une dette traîne trop longtemps, elle ne

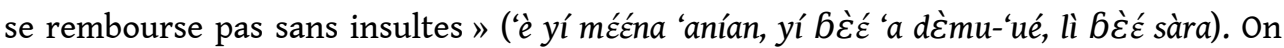
retrouve ici la forme la plus fréquente observée dans le discours proverbial, celle de l'implication en «si p, alors q"; cependant, peut-on considérer cette phrase comme un proverbe? Comme il l'a été dit plus haut au sujet de la commune désignation du proverbe et de la devinette en boomu, la dimension métaphorique, qui se joue au moment de l'énonciation, quand le sens du proverbe est appliqué à la situation vécue, est essentielle à la définition du " proverbe/wàwe » (Leguy, 2004). La formule ci-dessus reprend peut-être au proverbe sa forme pour donner un rythme au propos de l'énonciateur, mais si elle est énoncée au cours d'une conversation portant sur les 
dettes, elle ne peut pas être considérée comme un proverbe9. Ainsi que le disait Meschonnic, «Un proverbe n'est pas proverbe par la logique ou la structure qu'on y reconnait, car cette logique et cette structure se trouvent aussi ailleurs » $(1976,425)$.

On entend ainsi en boomu des énoncés qui semblent être proverbiaux dans leur formulation, mais qu'on hésite à désigner comme tels parce qu'on a l'habitude de les utiliser sans connotation, seulement pour leur faire dire ce qu'ils disent effectivement ${ }^{10}$. C'est le cas de toutes ces formules qui semblent être des maximes de bonne tenue et sont prononcées en guise de conseil en fonction des circonstances. Ainsi, un même énoncé pourra être considéré comme un proverbe dans certains cas et pas dans d'autres, selon l'usage qui en est fait. Si les constructions privilégiées relevées plus haut participent à la réussite d'une performance proverbiale, elles ne sauraient suffire à faire d'un énoncé un dire proverbial, au sens où l'entendent les Bwa. L'énoncé isolé de tout contexte perd sa dimension proverbiale, bien qu'il garde une « forme » qui pourrait permettre de le considérer comme un proverbe. Le rythme impliqué par les choix syntaxiques et la correspondance entre un énoncé et les structures récurrentes signalées plus haut ne suffisent pas. Manque une rupture au niveau sémantique, indispensable à la qualification d'un propos comme proverbial. À l'inverse, ne peut-on pas introduire une telle rupture sémantique sans pour autant rompre le rythme syntaxique du discours?

\section{Le recours à l'allusion proverbiale : le refus de rompre le rythme}

Il est d'autant plus important de relativiser l'importance accordée à l'aspect formel du proverbe que la façon dont le proverbe intervient dans le cours d'une conversation n'est pas toujours très respectueuse d'une forme codifiée retenue du proverbe. En effet, si le proverbe peut être connu sous une forme, ce n'est pas forcément sous cette forme canonique qu'il est utilisé. La citation sait s'adapter au contexte dans le monde de l'oralité.

Quand existe, dans une communauté linguistique, une grande connivence culturelle entre les gens, il peut être fait appel au proverbe sans recourir à la construction formelle sous laquelle on le reconnaît. On sait que certaines sociétés africaines ont ainsi développé un moyen de communication proverbial sans qu'il soit nécessaire de prononcer les mots des proverbes. Des objets ou quelques figures peintes ou dessinées suffisent à évoquer l'énoncé proverbial auquel on fait alors implicitement référence. Dans ce domaine, le cas bien connu des couvercles à proverbes sculptés de la province du Cabinda (RDC/Angola) est significatif (Vaz, 1976 ; Faïk-Nzuji, 1989). Chez les Bwa, on ne trouve pas de représentation plastique de proverbes utilisée dans la communication, mais il est courant de voir les bons diseurs de proverbes ne pas se donner la peine de prononcer les formules auxquelles ils font appel. Aimant préserver le mystère de leur parole, les locuteurs les plus âgés se refusent tout particulièrement à être trop explicites. Comment mieux voiler sa parole qu'en recourant à un proverbe obscur sur le mode de l'allusion? Connues et mémorisées, les formules se rappellent à l'esprit des interlocuteurs sans qu'il soit nécessaire de les énoncer, et jouent ainsi de manière tout aussi efficace que si elles étaient dites leur rôle dans l'interlocution.

D'après les événements de discours relevés en situation, notons qu'il peut être fait tacitement appel au proverbe de deux manières différentes. La première consiste à faire tout simplement allusion au raisonnement du proverbe, afin que ceux qui le 
connaissent se le remettent immédiatement en tête et appliquent sans peine son raisonnement non dit à la situation de sa non-émission. La formule proverbiale que les interlocuteurs entendent implicitement relève alors du sous-entendu. L'énonciateur sous-entend le proverbe, mais il dépend de la capacité qu'ont ses interlocuteurs à analyser la situation actuelle pour qu'il soit vraiment entendu, au sens de compris, par ceux-ci, le sous-entendu, comme le dit Ducrot, étant «laissé au "tu" » (1984, 20 et suivantes).

Prenons un exemple: une jeune fille était partie en ville pour se marier sans le consentement de sa famille, mais revint quelque temps plus tard, s'étant fâchée avec son mari. Mécontents de son comportement, ses parents ne lui firent pas bon accueil et le grand-père lui fit remarquer qu' «à présent, le bœuf égorgé faisait voir son sang ", faisant allusion au proverbe suivant: "On peut tuer un bœuf sans voir son sang jusqu'au moment où on le mange " (nùu wéè bwé nàa, 'a ló canu bèć le fá ló c⿱亠乂re). Ce proverbe de portée profonde ne sera pas dit à n'importe quelle occasion, tout comme on ne tue pas un bœuf sans une raison importante, des funérailles par exemple. Lorsqu'on s'apprête à tuer l'animal, on pense à l'événement festif, au bon repas que l'on va faire et au partage ; on ne pense pas au sang qu'il va verser. Ainsi n'envisage-t-on pas toujours toutes les conséquences de ce que l'on fait. La jeune fille désobéissante n'avait pas songé qu'un jour, elle voudrait revenir vers sa famille et que ses parents lui feraient alors sentir la désapprobation qu'elle n'avait pas voulu entendre auparavant. En prononçant ces mots, le grand-père annonçait que la pire conséquence de l'acte original était d'actualité. Son allusion faisait appel d'elle-même à la formule qu'il n'était nullement nécessaire d'énoncer pour que le proverbe se rappelle à l'esprit de la jeune fille et qu'elle comprenne, malgré l'obscurité de cet énoncé bien aimé des vieux, qu'on ne la laisserait pas agir impunément à l'encontre de l'avis familial. L'image du bœuf égorgé et du sang versé donne par ailleurs à cet énoncé une dose de tragique qui suffit à faire comprendre, à ceux pour qui le sens de l'émission resterait obscur, que la réplique n'est pas anodine.

La deuxième manière de faire allusion à un proverbe sans faire appel à sa forme canonique est l'utilisation d'une image issue d'un proverbe auquel on fait ainsi référence sans l'énoncer. Il s'agit alors de se saisir de l'image et de la traiter comme si elle était une simple image, mais en l'utilisant en tant que porteuse de l'histoire d'un proverbe bien précis que l'énonciataire devra connaitre s'il veut comprendre l'allusion et les propos de celui qui parle. On pourra par exemple évoquer seulement le sac en peau de chèvre (pui) pour faire allusion à la propension à mentir d'un interlocuteur, ce sac évoquant le proverbe suivant: «Ce qui est dans le sac "coince" le rapporteur » (bè ne mi puî, dàà sàbé jùn). "Montre ton sac " sera une manière de dire à celui qu'on soupçonne de mentir (ou de rapporter des faits dont il n'a pas été témoin, ce qui est équivalent pour les Bwa), qu'on n'est pas dupe de son jeu. Quand quelqu'un s'adresse à vous de pareille façon, on apprécie que vous puissiez entrer dans son jeu et répondre de la même manière, en faisant allusion à un autre énoncé proverbial, dans une subtile connivence d'amateurs de bonne parole. La conversation semble alors plate et anodine, aucune formule codifiée ne venant en rompre le tempo. Et pourtant, des proverbes sont bien échangés. D'un point de vue syntaxique en effet, rien ne vient signaler la présence du proverbe: pas de formule introductive annonçant le style indirect, pas de rythme binaire, pas de formulation condensée, pas de structure logique visible. Cependant, la 
rupture sémantique est bien présente, du moins pour ceux qui ont la compétence nécessaire à saisir implicitement le proverbe non énoncé.

Si la forme de l'énoncé joue un rôle dans sa mémorisation, en situation on ne prend pas toujours la peine de recourir à la formule codifiée lorsqu'on veut faire référence à un proverbe. Anscombre le dit bien lui-même :

je ne nie pas qu'il y ait des proverbes figés: comme toutes les catégories de la langue, celle des proverbes et autres formes sentencieuses possède des éléments figés. Ce que je refuse [...], c'est de considérer le figement comme un trait définitoire de la classe des proverbes.

(2005, en ligne, § 26).

Ainsi, les bons orateurs se jouent-ils des structures logiques et du rythme binaire, une allusion leur suffisant, en toute complicité, à recourir à un proverbe sans l'énoncer. La seule rupture introduite alors, à condition de comprendre ce qui est sous-entendu, est d'ordre sémantique.

Souvent retenus selon une formulation bien précise, parfois annoncés par une introduction, les énoncés proverbiaux sont reconnaissables lors de leur énonciation, entraînant une rupture du fait de leur rythme binaire ou bien de leur correspondance à certaines structures logiques propres à ce type de discours, en particulier à l'implication. Cependant si, face à un jeune interlocuteur qui doit être sermonné, il peut être utile de marquer son discours en imposant le rythme d'un énoncé bien frappé qui en renforce l'aspect sentencieux, entre amateurs de bonnes paroles, on pourra préférer « laisser entendre » et ne pas interrompre le flot ordinaire du discours. Le fait qu'il soit possible, voire valorisé par les bons diseurs de proverbes, de ne pas énoncer les formules en entier, et même d'y faire simplement allusion, conduit à se demander s'il n'y a pas alors, dans l'énonciation elle-même, une volonté de ne pas rompre le rythme, d'éviter le basculement, tout en ayant recours - même si c'est sur le mode allusif - au proverbe. Maîtrisé par les vieux adeptes du discours proverbial, le recours à l'allusion se présente comme une sorte de jeu, qui n'est pas très éloigné du jeu de devinette, où l'interlocuteur est invité à saisir la rupture sémantique en l'absence de toute rupture syntaxique. Ainsi, le discours proverbial peut-il s'émanciper des structures rythmiques par lesquelles on le reconnaît, dans la mesure où il peut se manifester subtilement en étant simplement sous-entendu, à condition que le corpus soit partagé par les interlocuteurs. Cette pratique ludique met l'accent sur le fait que dans le discours proverbial, la rupture sémantique importe plus que le changement de rythme, celui-ci n'en étant en quelque sorte qu'un outil de signalisation, un outil efficace, mais dont aiment à se dispenser les meilleurs diseurs de proverbes.

\section{BIBLIOGRAPHIE}

ANSCOMBRE, Jean-Claude (éd.), 2000, la Parole proverbiale, Langages, $\mathrm{n}^{\circ} 139$.

ANSCOMBRE, Jean-Claude, 2000, Paroles proverbiales et structures métriques, Langages, $\mathrm{n}^{\circ} 139$, p. 6-26. Persée, DOI:10.3406/lgge.2000.2377 
ANSCOMBRE, Jean-Claude, 2003, les Proverbes sont-ils des expressions figées ?, Cahiers de lexicologie, $\mathrm{n}^{\circ}$ 82, p. 159-173.

ANSCOMBRE, Jean-Claude, 2005, les Proverbes : un figement du deuxième type ?, Linx [En ligne], 53, Linx, DOI: 10.4000/linx.255

ANSCOMBRE, Jean-Claude, DARBOR, Bernard, ODDO, Alexandra (éds), 2012, La parole exemplaire. Introduction à une étude linguistique des proverbes, Paris, Armand Colin [coll. « Recherches »].

BARLEY, Nigel, 1972, A Structural Approach to the Proverb and maxim with Special Reference to the Anglo-Saxon Corpus, Proverbium, no 20, pp. 737-750.

CHAMBAT-HOUILLON, Marie-France et WALl, Anthony, 2004, Droit de citer, Rosny-sous-Bois, Bréal (Langages\&Co).

CONENNA, Mirella, 2000, Structure syntaxique des proverbes français et italiens, Langages, $\mathrm{n}^{\circ} 139$, p. 27-38, Persée, DOI:10.3406/lgge.2000.2378

DUCROT, Oswald, 1984, le Dire et le Dit, Paris, Minuit.

DUNDES, Alan, 1975, On the Structure of the Proverb, Proverbium, no. 25, pp. 961-973.

FAïK-NZUJI, Madiya C. (en collaboration avec Balila Babu), 1989, les Secrets de la parole sculptée. Lecture d'un taampha, couvercle à proverbes des Bawoyo (Zaïre/Angola), in Collectif, Graines de Parole, Paris, CNRS, p. 107-117.

GOMEZ-JORDANA FERARY, Sonia, 2012, le Proverbe : vers une définition linguistique. Étude sémantique des proverbes français et espagnols contemporains, Paris, L'Harmattan.

GOUVARD, Jean-Michel, 2006, Prolégomènes à une analyse métrique des proverbes, Cahiers de grammaire, $\mathrm{n}^{\circ}$ 30, p. 193-203.

GREIMAS, Algirdas Julien, 1970, Du sens, essais sémiotiques, Paris, Seuil.

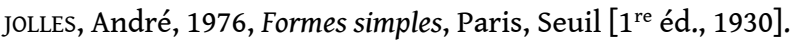

LEGUY, Cécile, 1996, Place du proverbe chez les Bwa du Mali. Étude ethnolinguistique, Thèse de doctorat en anthropologie sociale et ethnologie, Paris, EHEss.

LEGUY, Cécile, 2004, De la devinette au proverbe. Métamorphoses d'un genre, Cahiers de littérature orale, $\mathrm{n}^{\circ} 55$, p. 109-123.

LEGUY, Cécile, 2005, Formes et masques du discours proverbial, in Ursula Baumgardt et Abdellah Bounfour (éds), le Proverbe en Afrique : forme, fonction et sens, Paris, L'Harmattan/Inalco, p. 135-158. LEGUY, Cécile, 2006, Des noms cités dans les proverbes. Au sujet d'une modalité du reproche indirect en contexte africain, Cahiers de littérature orale, $\mathrm{n}^{\circ}$ 59-60, p. 165-183.

MESCHONNIC, Henri, 1976, les Proverbes, actes de discours, Revue des sciences humaines, t. XLI, $\mathrm{n}^{\circ} 163$, p. 419-430.

MESCHONNIC, Henri, 2009, Critique du rythme : anthropologie historique du langage, Lagrasse, Verdier

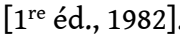

MILNER, George B., 1969, De l'armature des locutions proverbiales, essai de taxonomie sémantique, l'Homme, t. IX, nº 3, Paris, Mouton \& Co, p. 49-70.

PERMYAKOV, Grigorij L'vovic, 1979, From Proverb to Folk-tale. Notes on the general theory of cliché, Moscow, Nauka Publishing House - U.S.S.R. Academy of Sciences, "Studies in oriental folklore and mythology" [1 $1^{\text {re }}$ éd. en russe, 1970]. 
TAMBA, Irène, 2000, Formules et dire proverbial, Langages, $\mathrm{n}^{\circ}$ 139, p. 110-118.

VAZ, José Martins, 1976, les Couvercles sculptés des Cabindas, mabaia ma mzungu - Moyen de communication familiale. (Angola), Thèse de doctorat de troisième cycle, sous la direction de Paul

Mercier, Paris, Paris V-René Descartes/EHEss.

\section{NOTES}

1. Les Bwa (singulier Boo), appelés aussi Bwawa ou Bwaba au Burkina Faso, vivent dans une région de savane située à l'est du Mali et au sud-ouest du Burkina Faso. Ils parlent le boomu (ou bwamu), une langue gur. Les proverbes cités ici sont dans une variante dialectale du boomu majoritairement parlée sur le territoire boo malien, dite dialecte de Mandiakuy.

2. Le corpus de proverbes recueillis en situation d'énonciation a fait l'objet d'une thèse de doctorat (Leguy, 1996).

3. Par métrique, j'entends l'étude des proverbes en rapport à la versification, en particulier la mesure des longueurs, telle qu'a pu la faire ANSCOMBRE (2000) au sujet des proverbes français; cette étude est d'ailleurs critiquée par GOUVARD $(2006,197)$ qui propose lui-même une analyse prosodique fondée sur la métrique phonologique, plus adaptée à l'oral. Concernant les proverbes en boomu, langue fortement tonale, une telle étude supposerait une analyse phonologique et tonologique très précise, qui dépasserait le cadre de cet article.

4. Est appelé « énonciateur » celui qui produit l'énoncé en situation.

5. Il faut préciser que ces proverbes décomptés ici se retrouvent parfois dans les catégories précédemment mentionnées, puisque les énoncés émis par les personnages dotés pour l'occasion de la parole sont eux aussi des proverbes à part entière.

6. nìi-mána = 'un nì bèć mána : ma-part-nég.-n'existe pas = « ma part n'existe pas », « je ne suis pas concerné ».

7. Tu-si-vois-que-hyène-en train de-s'amuser-avec-chèvres-petit-têtes-alors-chosessont-faites.

8. Tu-si-vois-que-hyène-en train de-s'amuser-avec-chèvres-petit-têtes-alorselles + démonstratif-se trouvent-son-ventre-nombre-pluriel-n'existe pas.

9. Voir à ce sujet la différence qu'ANSCOMBRE $(2000,10-12)$ fait entre les phrases Lsentencieuses et les phrases ON-sentencieuses.

10. On peut également rapprocher cette conception du dire proverbial de ce que dit GREIMAS au sujet de la différence entre proverbes et dictons (1970, 309-314). 


\section{RÉSUMÉS}

Le dire proverbial présente un aspect formel souvent assez particulier qui fait sa spécificité comme genre dit «figé » de la littérature orale. À partir d'un corpus de cinq cents proverbes recueillis auprès des Bwa du Mali, il est possible de déterminer des formes récurrentes et un rythme propre au proverbe. Cependant, en situation d'énonciation, ce n'est pas toujours la forme qui fait le proverbe, celui-ci pouvant ne pas être prononcé intégralement, être tronqué ou même seulement évoqué par un mot ou quelques tons chantonnés entre les lèvres. Peut-on alors véritablement parler de rythme proverbial ? Faut-il nécessairement qu'un basculement ait lieu dans l'énonciation pour qu'on sache qu'un proverbe a été émis? Dans quelle mesure le rythme fait-il le proverbe?

A proverbial saying usually presents specific formal aspects that constitute it as a special "set" genre within oral literature. From a corpus of five hundred proverbs collected from the Bwa of Mali, it is possible to identify recurring patterns of forms and rhythms. When used in speaking situations, however, this patterning is not always upheld. Proverbs can indeed be delivered in part: they can be truncated, mentioned by a single word, or a few tones hummed between the lips. In what respect can one then speak of a proverbial rhythm? How do we know that in the course of speaking a proverb is being stated? To what extent does rhythmical patterning make the proverb?

\section{INDEX}

nomsmotscles Bwa

Thèmes : littérature orale

Index géographique : Mali

Mots-clés : implication, proverbe, énonciation

Keywords : Involvement, Rhythm, Enunciation, Proverb, Bwa (Mali)

\section{AUTEUR}

\section{CÉCILE LEGUY}

Université Sorbonne Nouvelle-Paris 3 - LACITO-CNRS (UMR 7107) 